number exceeds the present accepted safety levels at cord and breast milk samples. Preventing ETS, limiting fish consumption and improved living conditions for pregnant women may decrease exposure levels.

\section{PELOD-2: AN UPDATE OF THE PEDIATRIC LOGISTIC ORGAN DYSFUNCTION SCORE}

doi:10.1136/archdischild-2012-302724.0099

1,2S Leteurtre, ${ }^{2,3} \mathrm{~A}$ Duhamel, ${ }^{2,3} \mathrm{~J}$ Salleron, ${ }^{2,4} \mathrm{~B}$ Grandbastien, ${ }^{5} \mathrm{~J}$ Lacroix, ${ }^{1,2} \mathrm{~F}$ Leclerc, on behalf of the Groupe Francophone de Réanimation et d'Urgences Pédiatriques (GFRUP). ${ }^{1}$ Pediatric Intensive Care Unit, Jeanne de Flandre University Hospital; 'EA2694, Univ Lille Nord de France, UDSL; ${ }^{3}$ Department of Biostatistics, CERIM, University of Medicine; ${ }^{4}$ Department of Epidemiology and Public Health, Calmette Hospital, Lille, France, ${ }^{5}$ Pediatric Intensive Care Unit, Sainte-Justine Hospital, Université de Montréal, Montréal, OC, Canada

Background and aims Organ dysfunction scores, such as the PEdiatric Logistic Organ Dysfunction (PELOD) score developed in 1999 , are primarily designed to describe the severity of organ dysfunction. This study was undertaken to update and improve the PELOD score, using a larger and more recent dataset.

Methods We did a prospective, observational, multicentre cohort study in nine French-speaking multidisciplinary, tertiary-care PICUs of university-affiliated hospitals between June 2006 and October 2007. We collected data on variables considered for the PELOD-2 score at seven time-points after PICU admission: days 1 , $2,5,8,12,16$ and 18, plus PICU discharge. For each variable, the most abnormal value observed during each time point was collected. Identification of the best variable cutoffs was performed using bivariate, multivariate regressions and bootstrap process. The outcome was vital status at PICU discharge. We used area under receiver operating characteristic curve (AUC) to evaluate discrimination and Hosmer-Lemeshow goodness-of-fit test to evaluate calibration

Results We included 3671 consecutive patients (median age 15.5 months IOR 2.2-70.7). Mortality rate was 6.0\% (222 deaths). Discrimination and calibration of the PELOD 2 score were 0.93 and $9.31(\mathrm{p}=0.317)$ respectively.

Conclusion We developed and validated the PELOD-2 score, which allows assessment of the severity of cases of MODS in PICU with a continuous scale. The score will be in the public domain, which means that it can be freely used in clinical trials.

\section{NEONATAL DISEASE SEVERITY SCORES AND THEIR PREDICTIVE VALUE 3FOR EARLY MORTALITY: A POPULATION-BASED STUDY ON SUBGROUPS OF VLBW INFANTS}

doi:10.1136/archdischild-2012-302724.0100

${ }^{1} \mathrm{R}$ Hentschel, ${ }^{1} \mathrm{~K}$ Günther, ${ }^{2} \mathrm{~W}$ Vach, ${ }^{3} \mathrm{M}$ Teufel, ${ }^{4} \mathrm{~T}$ Böhler, ${ }^{5} \mathrm{M}$ Mohrmann, ${ }^{6} \mathrm{~J}$ Arand, ㄱ Dick, ${ }^{8} \mid$ Bruder, ${ }^{9} \mathrm{~W}$ Kachel. ${ }^{1}$ Neonatology/Intensive Care, Center for Pediatrics and Adolescent Medicine University of Freiburg; '2Div. Clinical Epidemiology, Institute of Medical Biometry and Medical Informatics, University Medical Center Freiburg, Freiburg im Breisgau; ' ${ }^{2}$ ept. Pediatrics, Academic Hospital Sindelfingen-Böblingen, Böblingen; ${ }^{4}$ Medical Service of Statutory Health Insurance Companies (MDK) Baden-Württemberg, Karlsruhe; ${ }^{5}$ Medical Service of Statutory Health Insurance Companies (MDK) BadenWürttemberg, Lahr; ${ }^{6}$ Div. Neonatology, Univ. Children's Hospital Tübingen, Tuebingen; ${ }^{7}$ Registry of Neonatal Quality Assurance Program, Baden-Württemberg Chamber of Physicians; ${ }^{8}$ Registry of Baden-Württemberg Hospital Quality Assurance (GeQiK), Stuttgart; ${ }^{9}$ Dept. Pediatrics, Academic Hospital Heilbronn, Heilbronn, Germany

Background and aims Benchmarking of newborn mortality needs risk-adjustment of data for heterogeneous sub-populations. To assess utility of neonatal disease severity scores CRIB, CRIB-II and PREM and impact of influenceable items ( $\mathrm{FiO}_{2} \mathrm{max}, \mathrm{FiO}_{2} \mathrm{~min}$, body temperature (BT) base excess $(\mathrm{BE}))$ to predict mortality in VLBW infants $(V L B W)$, ELBW infants < 750g (BW750), g.a. 22-25 weeks (GA22-25).

Methods Analysis of birth cohorts of years 2003-2008 from the Baden-Württemberg registry. Inclusion criteria: GA $<33$ weeks and $\mathrm{BW}<1.500 \mathrm{~g}$. Variables considered: $\mathrm{GA} ; \mathrm{BW}$; gender; $\mathrm{BT} ; \mathrm{FiO}_{2} \max ;$ $\mathrm{FiO}_{2}$ min; BE; malformation; death. Calculation of standard CRIB CRIB-II and PREM with/without omission of selective items. Calculation of predictive value of scores/subscores for whole cohort $V L B W$, subgroups BW750 and GA22-25 using AUC of ROC curves. Wilcoxon/Mann-Whitney U-test, Fishers exact test, Pearson-ChiSquare test.

Results Total of 5.340 cases, 862 cases $<750 \mathrm{~g}$. AUC for $V L B W /$ BW750: CRIB $0.89 * / 0.77$, CRIB-II $0.86 * / 0.78$, PREM $0.86 * / 0.77$ ( $\left.{ }^{*} \mathrm{p}<0.01\right)$. For GA22-25 AUC of CRIB/PREM was 0.80/0.70. Lower AUC of all 3 modified scores without BT and/or BE, for instance PREM $=0.82$ (VLBW) and 0.73 (BW750). AUC of CRIB without influenceable parameters dropped for $V L B W$ from 0.89 to 0.81 , for $B W 750$ from 0.77 to 0.66 (compared to modified CRIB-II=0.71, modified PREM=0.73).

Conclusions Standard CRIB is superior to standard CRIB-II, standard PREM, and all score modifications without influenceable items. No difference exists between the 3 scores when omitting influenceable parameters. For ELBW infants $<750 \mathrm{~g}$ all standard scores are equally predictive, but without influenceable parameters AUC of CRIB is inferior to that of CRIB-II or PREM.

\section{FETAL NUTRITION-WHAT CAN WE LEARN TO BETTER NOURISH THE PRETERM INFANT?}

doi:10.1136/archdischild-2012-302724.010

WW Hay. Department of Pediatrics, University of Colorado School of Medicine, Aurora, CO, USA

Most preterm infants fail to grow after birth and end up growth restricted by term. The main reason is inadequate nutrition. From studies of normal fetal growth and development, we have gained important insight into the requirements for such growth and development that could be applied to the preterm infant of the same preterm gestational age. Maintaining normal blood oxygen content values to support the high rates of cellular metabolism and protein synthesis is essential to promote normal rates of growth Glucose should be supplied at rates that maintain normal fetal glucose concentrations. Normal human fetal development involves considerable body fat deposition, but more emphasis should be placed on providing essential fatty acids to promote membrane development in neural tissue. Amino acid utilization rates based on fetal animal growth data, when scaled to human fetal growth rates, predict amino acid requirements of $3.6-4.8 \mathrm{~g} / / \mathrm{kg} /$ day at $\sim 24-30$ weeks gestation. There is a linear correlation between amino acid supply to preterm infants and protein balance, at least through $3 \mathrm{~g} / \mathrm{kg} /$ day. While energy is required for protein synthesis, above 80-90 non-protein $\mathrm{kcal} / \mathrm{kg} / \mathrm{d}$, there is no further increase in protein gain for an increase in energy intake. Improved protein and energy intake in preterm infants that more closely matches fetal nutrition is associated with improved brain growth and neurocognitive outcomes. Insulin concentrations that result from such nutrition probably are sufficient for normal growth insulin infusions do not add more to promote growth than increased amino acid/protein nutrition and produce significant adverse effects.

\section{RANDOMIZED, CONTROLLED TRIAL OF SLOW VERSUS RAPID ENTERAL FEEDING ADVANCEMENTS ON THE CLINICAL OUTCOMES OF PRETERM INFANTS 750-1250G}

doi:10.1136/archdischild-2012-302724.0102 